\title{
Study Protocol for a Randomised Controlled Trial to Investigate the Enhancement of Diabetic Foot Ulcer Healing Using Low-Magnitude High-Frequency Vibration Treatment
}

\section{Samuel Ka Kin Ling ( $\nabla$ samuel-kk-ling@alumni.cuhk.net )}

Chinese University of Hong Kong https://orcid.org/0000-0003-4528-9074

Natalie Cheuk Lam Hung

The Chinese University of Hong Kong

Wing-Hoi Cheung

Chinese University of Hong Kong

Patrick Shu-Hang Yung

Chinese University of Hong Kong

\section{Research Article}

Keywords: Diabetic Foot, Whole Body Vibration, Low Magnitude High Frequency Vibration

Posted Date: June 23rd, 2021

DOI: https://doi.org/10.21203/rs.3.rs-221851/v1

License: (c) (1) This work is licensed under a Creative Commons Attribution 4.0 International License.

Read Full License 
Title

2 Study Protocol for a Randomised Controlled Trial to Investigate the Enhancement of Diabetic Foot Ulcer Healing Using Low-Magnitude High-Frequency Vibration Treatment

4

5

\section{Names protocol contributors}

Samuel Ka-Kin LING

samuel-kk-ling@alumni.cuhk.net

Natalie Cheuk-Lam HUNG

nataliehung@cuhk.edu.hk

Louis Wing-Hoi CHEUNG

louischeung@cuhk.edu.hk

Patrick Shu-Hang YUNG

patrickyung@cuhk.edu.hk

Corresponding Author:

Samuel Ka-Kin LING

MBChB, ChM, FHKCOS, FRCSEd, FHKAM

samuel-kk-ling@alumni.cuhk.net

Clinical Assistant Professor

Abstract

Dept of Orthopaedics and Traumatology, Faculty of Medicine, CUHK

Dept of Orthopaedics and Traumatology, Faculty of Medicine, CUHK

Dept of Orthopaedics and Traumatology, Faculty of Medicine, CUHK

Dept of Orthopaedics and Traumatology, Faculty of Medicine, CUHK

Dept of Orthopaedics and Traumatology, Faculty of Medicine, CUHK

5/F, LWC Clinical Sciences Building, Prince of Wales Hospital, Shatin, NT, Hong Kong SAR 
Background:

32 Diabetes has a prevalence of $11.6 \%$ in China with diabetic foot ulcerations affecting over 30 million Chinese.

$3385 \%$ of these patients require amputation and 5 -year mortality for diabetics is $70 \%$ when associated foot ulcers.

34 Clinical trials have shown that standing on whole-body vibration platforms, specifically low-magnitude high-

35 frequency vibration (LMHFV); promotes angiogenesis, enhances muscle bulk and accelerates epithelization.

36 Investigation on diabetic rats with foot wounds found accelerated wound healing, increased perfusion and 37 upregulation of factors such as VEGF, PECAM-1 and PCNA. Hypothesis: We postulate LMHFV will enhance 38 diabetic foot ulcer healing.

Methods:

41 Prospective, single-centre, randomised control trial to treat 106 subjects with diabetic foot ulcers. Interventions: The intervention group will stand on LMHFV whole-body vibration platforms for $20 \mathrm{~min}$ on alternate days for 20 weeks, together with conventional dressing by a trained wound-care nurse as in the control group.

45 Main Outcome Measures: Ulcer size will be measured at multiple time points, the incidence of amputations/infections will be recorded, perfusion via ankle-brachial pressure index will be calculated and foot function via the foot and ankle outcome score will be analysed.

Data analysis: Repeated measure of ANOVA to analyze time-point differences and student's t-test for same time-point comparison.

51 Discussion:

52 This is the first clinical trial to investigate the effect of whole-body vibration on diabetic foot ulcers. It will show 53 us if the results from animal studies will translate into clinically significant results. If positive effects are established, whole-body vibration can be a valuable treatment regime to tackle diabetic foot ulcers.

\section{Keywords}

60 Diabetic Foot, Whole Body Vibration, Low Magnitude High Frequency Vibration, 


\section{Administrative information}

63 'Note: the numbers in curly brackets in this protocol refer to SPIRIT checklist item numbers. The order of the

64 items has been modified to group similar items (see http://www.equator-network.org/reporting-

65 guidelines/spirit-2013-statement-defining-standard-protocol-items-for-clinical-trials/)

\begin{tabular}{|c|c|}
\hline Title $\{1\}$ & $\begin{array}{l}\text { Study Protocol for a Randomised Controlled Trial to Investigate the } \\
\text { Enhancement of Diabetic Foot Ulcer Healing Using Low-Magnitude } \\
\text { High-Frequency Vibration Treatment }\end{array}$ \\
\hline Trial registration $\{2 \mathrm{a}$ and $2 \mathrm{~b}\}$. & Trial registration: NCT04275804 clinicaltrials.gov (19 Feb 2020) \\
\hline Protocol version $\{3\}$ & Version 01 (19 Feb 2020) \\
\hline Funding $\{4\}$ & The project has no external funding source. \\
\hline Author details $\{5 a\}$ & $\begin{array}{l}\text { Samuel Ka-Kin LING } \\
\text { samuel-kk-ling@alumni.cuhk.net } \\
\text { Dept of Orthopaedics and Traumatology, Faculty of Medicine, CUHK } \\
\text { Natalie Cheuk-Lam HUNG } \\
\text { nataliehung@cuhk.edu.hk } \\
\text { Dept of Orthopaedics and Traumatology, Faculty of Medicine, CUHK } \\
\text { Louis Wing-Hoi CHEUNG } \\
\text { louischeung@cuhk.edu.hk } \\
\text { Dept of Orthopaedics and Traumatology, Faculty of Medicine, CUHK } \\
\text { Patrick Shu-Hang YUNG } \\
\text { patrickyung@cuhk.edu.hk } \\
\text { Dept of Orthopaedics and Traumatology, Faculty of Medicine, CUHK }\end{array}$ \\
\hline
\end{tabular}




\begin{tabular}{|l|l|}
\hline $\begin{array}{l}\text { Name and contact information for } \\
\text { the trial sponsor }\{5 b\}\end{array}$ & n/a there is no trial sponsor \\
\hline Role of sponsor $\{5 c\}$ & $n / a$ there is no sponsor \\
\hline
\end{tabular}

69 Introduction

\section{Background and rationale $\{6 \mathbf{a}\}$}

71 Diabetic Foot Ulcers are a major problem.

72 Diabetes is a major non-communicable disease which affects has a prevalence of $11.6 \%$ in the Chinese, affecting over 110 million people. (1-3). Diabetics have more than doubled in our locality over the past 20 years, this has been attributed to many different factors including urbanization, genomic predisposition, dietary (rice is high in carbohydrates) and the generally sedentary lifestyles adopted in Hong Kong. (4-7) It is reported that $30 \%$ of diabetics have foot ulceration in which $\sim 60 \%$ are non-healing. $(8,9)$ This creates a huge social-economic burden in society and utilizes 184 hospital bed days/1000 diabetic patients/year. (10)

Amputations are common.

Studies have reported that $20-85 \%$ of patients with diabetic foot ulceration eventually require an amputation $(8,9)$ and $70 \%$ of them die within 5 years. This is significantly higher (x 2.5 times) than diabetic patients without foot ulcerations. $(3,8,11,12)$ There are many confounding factors that contribute to the high mortality with decreased ambulation (or prolonged bed-rest) due to amputations being one of them. The aetiology is multifactorial with neuropathy, vasculopathy and decreased muscle bulk being the major factors. $(8,11)$ Current conventional treatment is surgical debridement in conjunction with revascularization and proper glycaemic control. $(10,13)$ However, unlike biophysical interventions such as whole-body vibration $(14,15)$, they are often less effective in improving the microcirculation and intrinsic foot muscle bulk. Limitations of conventional dressing are indirectly evidenced by the high amputation rates $(13,16-18)$ and the many ongoing studies to find a better solution to this widespread problem. Different modes of wound dressing are currently being investigated such as artificial skin and biologically augmented dressing material (e.g stem cells, concentrated platelets etc.) but most are one-off expensive interventions.

Whole-body vibration can modulate the healing response at a cellular level. Whole-body vibration platforms (fig 01 and fig 02 in supplementary notes) are widely used by the public for exercise and weight loss regimes; there are two main types, the low-magnitude high-frequency vibration (LMHFV) platforms which vibrates in the vertical axis with a mild displacement (max $0.3 \mathrm{~g}$, displacement $<0.1 \mathrm{~mm}$ ) and the side-to-side vibration platforms which have a larger displacement (max 22g, displacement 
$12.2 \mathrm{~mm}$ ). They are a relatively cheap, non-invasive biophysical intervention that vibrates the body when the subject is standing on top.

100 Previous clinical trials on whole-body vibration have found enhancement of healing and modulation of 101 various hormonal factors for bone and muscles. (19-21) An RCT involving 710 participants showed that therapy with a Low-Magnitude High-Frequency Vibration platform ( $20 \mathrm{~min} /$ day 5 days/ week) significantly improved muscle strength, less bone loss and improved quality of life. (14) Over the years, clinical trials using low-magnitude high-frequency vibration platforms (LMHFV) have shown that it is a safe and effective treatment modality.

Vibration enhances DM ulcer healing.

Animal studies on low-magnitude high-frequency vibration showed improved circulation evaluated by doppler USG, micro-CT angiography and histology in a rat model. There was increased angiogenesis and expression of vascular endothelial growth factor (VEGF) as well as improved bone formation and mineralization. A study of 96 diabetic rats with foot wounds showed that LMHFV significantly accelerated wound healing and improved skin micro-circulation with upregulation of various healing markers. $(15,22)$ The wound size was significantly smaller, blood glucose levels were significantly lower and glucose transporter 4 expression (GLUT 4 immunoblotting) was significantly better in the vibration group. Perfusion in the wound, measured using laser Doppler, was also significantly better in the vibration group and more granulation and epithelial tissue were seen on histology. Platelet endothelial cell adhesion molecule-1 (PECAM-1), a signalling molecule that upregulates angiogenesis and endothelialization, was significantly higher in the vibration group. Proliferating cell nuclear antigen was also higher which signifies there was more cell proliferation in the vibration group. Vascular Endothelial Growth Factor (VEGF), which promotes bone formation, haematopoiesis, wound healing as well as angiogenesis was significantly elevated in the vibration group compared to the controls.

Clinical trials $(14,20)$ using low-magnitude high-frequency vibration (LMHFV) show that it is a safe intervention for human subjects, and laboratory findings on DM ulcer healing suggest that there is evidence whole-body vibration may enhance diabetic ulcer healing in our patients.

\section{Objectives $\{7\}$}

We postulate that Low-Magnitude High-Frequency Vibration (LMHFV) will enhance diabetic foot ulcer healing and decrease the need for major amputation.

\section{Trial design $\{8\}$}

131 Single Blind, Parallel group, 1:1 allocation, Superiority, Single centre, Randomised Controlled Trial.

\section{Methods: Participants, interventions and outcomes}

135 Study setting $\{9\}$ 
136 Participants will be recruited from the patients in the Orthopaedic \& Traumatology department at a tertiary

137 teaching hospital affiliated to CUHK.

\section{Eligibility criteria $\{10\}$}

140 Inclusion criteria:

$141 \quad>18$ years old (legally able to self-sign consent)

142 - Able to stand independently

143 . Biochemically confirmed Diabetes with a fasting plasma glucose $\geq 7.0 \mathrm{mmol} / \mathrm{L}$, or a random plasma

144 glucose $\geq 11.1 \mathrm{mmol} / \mathrm{L}$ or hemoglobin $\mathrm{A} 1 \mathrm{c}(\mathrm{HbA} 1 \mathrm{c})$ level $\geq 6.5 \%$

145 Ulcers will be below the level of the malleoli, excluding those confined to the interdigital web space

146 - Cross-sectional area of the index ulcer should be $50-1000 \mathrm{~mm} 2$

147 - Wagner stage 2-3

148 - Not active infection according to the Infectious Diseases Society of America guidelines

150 Exclusion criteria:

151 - Severe cognitive impairment or severe comorbidity, which may impair the ability to adhere to

152 intervention plan, e.g. severe dementia, poor cardiopulmonary reserve requiring home oxygen, daily

153 hemodialysis etc.

154 - Evidence of active infection

155 - Recent revascularization procedure ( $<12$ weeks)

156 - Recently received medication/intervention which might affect cell proliferation (eg chemotherapy,

157 radiotherapy etc)

158 - Allergy to dressing, adhesives or antibiotics

159 Incapable to understand the study protocol or provide written informed consent

160 - Presence of other foot deformities

161

Who will take informed consent? $\{26 \mathrm{a}\}$

163 Principal Investigator/ Research Assistant will obtain written consent from all participants.

165 Additional consent provisions for collection and use of participant data and 166 biological specimens $\{26 \mathrm{~b}\}$

167 There are no plans for participant data to be used in ancillary studies

\section{Interventions}

\section{Explanation for the choice of comparators $\{6 \mathrm{~b}\}$}

171 We did not include a sham vibration group because it is obvious to the participants if the vibration is present 172 or not. For our previous investigations, we have attempted to ask participants to stand on a sham platform 
173 for 20mins, but compliance was low. In some muscle studies, the control group can perform different

174 exercises on a platform for 20 mins, however there are no proven exercise regimes that help diabetic foot

175 ulcer healing

Control group: conventional dressing

- Alternate day dressing in a designated clinic by a trained nurse specialized in wound care

- Dressing by a specialized wound-nurse is the current gold-stand of treatment for diabetic ulcers (13).

- We did not include a sham vibration group because it is obvious to the participants if the vibration is present or not. For our previous investigations, we have attempted to ask participants to stand on a sham platform for 20 mins, but compliance was low. In some muscle studies, the control group can perform different exercises on a platform

- Alternate day dressing in a designated clinic by a trained nurse specialized in wound care

- The duration of dressing will last until participant's wound is healed. If it is not healed after the study period, PI will look into the case to see if other treatments are needed.

- Alternate day $20 \mathrm{~min}$ sessions on a self-designed vibration platform with lowmagnitude high-frequency vibration $(35 \mathrm{~Hz}, 0.3 \mathrm{~g}$ peak-to-peak displacement $<0.1 \mathrm{~mm}$ ). Since the participants will return for dressing change on alternate days, the vibration group will also undergo the LMHFV during the same attendance.

- Our previous study have shown that the dosage of LMHW at $20 \mathrm{~min} / \mathrm{session} 3$ days/week is sufficient. (14)

- 10 Low-Magnitude High-Frequency vibration platforms are available in our outpatient department at Prince of Wales Hospital; these machines are available to be used by 
our patients. In addition, 150 community centres distributed around New Territories, Kowloon and HK Island are equipped with our LMHFV machines. They are open to the public either free-of-charge or with a nominal fee. Participants will be equipped with a personal NFC smart card that will log and record their usage of the platforms.

\section{Criteria for discontinuing or modifying allocated interventions $\{11 b\}$}

Interim data analysis will be performed at 20weeks, the investigators will then decide on discontinuing/modifying the intervention based on the judgement of safety and benefits. Participant may experience mild discomfort including itchiness and redness of the lower limb skin. The symptoms should resolve shortly after intervention. If these symptoms do not resolve/worsen after 30 minutes, it should be reported as an adverse event. PI will then look into the case and decide if the participant is suitable to continue the intervention for the rest of the study period.

\section{Strategies to improve adherence to interventions $\{11 \mathrm{c}\}$}

- Conventional dressing: Each attendance to wound dressing will be recorded by the clinic nurse.

- LMHFV Vibration: each participant in the vibration intervention group will be assigned a personal 'smart card'. This smart card will record each utilization of the vibration platform to

\section{Relevant concomitant care permitted or prohibited during the trial $\{11 \mathrm{~d}\}$}

The use of LMHFV vibration will not require alteration to usual care pathways (including use of any medication) and these will continue for both trial arms.

\section{Provisions for post-trial care $\{30\}$}

There is no anticipated harm and compensation for trial participation. Usual care pathways will be provided post-trial.

Outcomes $\{12\}$

\section{Primary Outcome:}

- Ulcer size: 0wk, 2wk, 8wk, 14wk, 20wk, 52wk

- The baseline ulcer size will be measured at 0 weeks and a core interim measurement will be conducted at 20 weeks since $30 \%$ of ulcerations will heal at 20 weeks using conventional dressing. (13) A 1-year reassessment will help differentiate if the 20-week course of LMHFV therapy has created sustainable changes. Multi-ulcer will not be considered. 
The early follow-up at 2 weeks will serve as a chance for the participant to voice out difficulties and for the investigator to provide early intervention if required. Subsequent 6-weekly documentation of the change in ulcer size will help decipher the ideal duration of LMHFV therapy.

\section{Secondary Outcome:}

\section{Time (days) to healing}

- The day of complete wound closure will be documented during their alternate day dressings in the designated clinic. The participant will still be reviewed at 20 weeks and 1 year for the primary outcome.

- Mean of time to healing will be recorded.

\section{Incidence of amputation}

- The reason and day of below knee/above knee amputation will be recorded. Details will be retrieved from the Clinical Management System, the centralized electronic healthcare system utilized by public hospitals in Hong Kong.

3. Incidence of secondary infection: Defined by IDSA criteria of infection (23)

- The number of hospitalized days and number of days of systemic antibiotic therapy will be documented. Details will be retrieved from the Clinical Management System, the centralized electronic healthcare system utilized by public hospitals in Hong Kong.

4. Perfusion via the Ankle-brachial pressure index (ABI): 0wk, 20wk

- The measurement of the ABI will be standardized. (24) The subject will be at rest in supine position for 10mins, Doppler ultrasound to measure systolic blood pressure twice in the posterior tibial artery (If there is no obtainable signal in the posterior tibial artery, the dorsalis pedis will be used.), the average systolic blood pressure in the posterior tibial artery/dorsalis pedis divided by the higher of the SBP in the two arms will be used to calculate the ABI. (The higher SBP of the arm will be used in these calculations due to previous studies showing a strong association between peripheral arterial disease and subclavian stenosis)

5. Foot function via the Foot and Ankle Outcome Score (FAOS) (25): 0wk, 20wk

- Foot function will be measured using the FAOS which is a variant of the Knee Injury and Osteoarthritis Outcome Score designed specifically for problems related to the foot and ankle region. The FAOS also has validated translations in English and Chinese; it is a questionnaire consisting of 5 Likert score subscales; Pain, Symptoms, ADL, Recreation and Foot related QOL. Answers are graded a score from 0-4 and a normalised score can be calculated for each subscale (100 is asymptomatic while 0 indicates extreme symptoms).

\section{Participant timeline $\{13\}$}


Consultation at

Orthopaedics OPD

-Suffering from DM Foot Ulcers

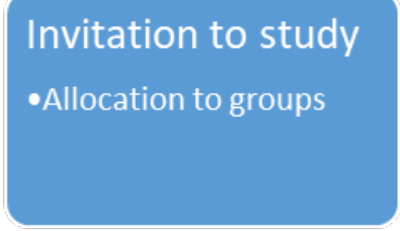

Baseline

Assessment

Follow-up

assessments
Interventions

\begin{tabular}{|c|c|c|c|c|c|c|c|c|c|c|c|c|c|}
\hline \multicolumn{10}{|c|}{ Timeline: } \\
\multicolumn{10}{|c|}{ Study Protocol for a Randomised Controlled Trial to Investigate the Enhancement of Diabetic Foot Ulcer } \\
Healing Using Low-Magnitude High-Frequency Vibration Treatment \\
\hline \\
\hline
\end{tabular}

\section{Sample size $\{14\}$}

284106 subjects randomized 1:1 into the two treatment groups.

The sample size was calculated by using the primary endpoint of wound size in G*Power 3.1.9.4 (Germany). Using a 1:1 randomization ratio a type I error rate (a-level) of 0.05 and power of 0.95 . Our vibration study of diabetic rat wounds showed an effect size $d$ of 0.99 . We estimate a tuned-down effect in human subjects, thus using an effect size of 0.7 we calculated that we should recruit 90 participants with 45 in each group. (22) To account for a dropout rate of $15 \%$, we will recruit 106 participants. 


\section{Recruitment $\{15\}$}

Participants will be recruited from the patients in the Orthopaedic \& Traumatology department at the Prince of Wales Hospital, the tertiary teaching hospital affiliated to CUHK. All patients from the Orthopaedic and Traumatology department will be screened. Those who meet the inclusion and exclusion criteria will be recruited.

To ensure sufficient recruitment, patients can be recruited from affiliated hospitals such as Alice Ho Miu Ling

\section{Assignment of interventions: allocation}

\section{Sequence generation $\{16 a\}$}

304 There is no list of factors for stratification. Permuted block randomization is used.

305 Generation of the allocation sequence: Research Assistant

\section{Concealment mechanism \{16b\}}

308 Sequentially numbered. The opaque envelopes containing the treatment allocation are sequentially

309 numbered and sealed. They are opened by the principal investigator on participant enrolment.

\section{Implementation $\{\mathbf{1 6 c}\}$}

312 Enrollment of participants: Principal Investigator

313 Assignment of participants to interventions: Principal Investigator or research assistant

314 Allocation sequence: Principal Investigator or research assistant

\section{Assignment of interventions: Blinding}

\section{Who will be blinded $\{17 \mathrm{a}\}$}

319 Single Blinded. Participants will know they are undergoing vibration therapy, but the assessors and data 320 analysts will be blinded.

323 The design is open label with only outcome assessors being blinded so unblinding will not occur. 


\section{Plans for assessment and collection of outcomes $\{18 \mathrm{a}\}$}

All collected personal data and medical information relevant to this study about the subjects will be strictly confidential. The data can be accessed only by the principal investigator, co-investigators and the research assistant(s) in charge. Subjects will only be identified by a study number and initials in the study database, and no personal identity will be disclosed when study results are being reported and/or published. The data will be kept for an additional 5 years for monitoring. Data that are related to future patient management will be stored in hospital CMS system.

\section{Plans to promote participant retention and complete follow-up \{18b\}} $\mathrm{n} / \mathrm{a}$ all participants are chronic disease patients with regular scheduled follow-up regardless of this trial.

\section{Data management $\{19\}$}

The nurse and research assistant (both reporting directly to the principal investigator) will be responsible for data collection and data entry while the principal investigator will be responsible for data analysis. The principal investigator himself will make decisions to terminate the trail trial if needed. Data quality is ensured by double data entry (the nurse and the research assistant). Electronic data entry will be used.

\section{Confidentiality $\{27\}$}

343 All collected personal data and medical information relevant to this study about the subjects will be strictly confidential. Subjects will only be identified by a study number and initials in the study database, and no personal identity will be disclosed when study results are being reported and/or published. Only the trial team including principal investigator and research assistant will have the right to access the data. genetic or molecular analysis in this trial/future use $\{33\}$

\section{Statistical methods}

\section{Statistical methods for primary and secondary outcomes $\{20 \mathrm{a}\}$}

Intention to treat population will be used. Repeated measures of ANOVA will be used to analyze the ulcer size groups and time points differences with be analyzed with post-hoc Bonferroni tests. Student's t-test for two independent samples will be used to compare groups of the same time point. Non-parametric tests will be used if data are not normally distributeThe Kruskal-Wallies test and Matt-Whitney $U$ test will be used 
instead of ANOVA and t-tests in case the data are not normally distributed. A mixed-effect model will be used to analyse the primary outcome. ANOVA will be used for analysing the secondary outcomes. Statistical analyses will be performed using IBM SPSS 25 (IBM, Armonk, NY, USA), and statistical significance was considered at $p<0.05$. This protocol will follow the CONSORT 2010 statement (BMC Medicine).

\section{Interim analyses $\{21 b\}$}

PI will monitor the results and make final decisions. An interim analysis will be performed with week 20 data. Ulcer size, time to healing, incidence of amputation, incidence of secondary infection, perfusion and foot function will be used in the interim analysis.

\section{Methods for additional analyses (e.g. subgroup analyses) $\{20 \mathrm{~b}\}$}

$\mathrm{n} / \mathrm{a}$, there are not current plans for subgroup analysis.

Methods in analysis to handle protocol non-adherence and any statistical methods to handle missing data $\{20 \mathrm{c}\}$

Missing data will be accounted for via listwise deletion.

\section{Plans to give access to the full protocol, participant level-data and statistical code} $\{31 c\}$

The datasets analysed during the current study are available from the corresponding author on reasonable request.

\section{Oversight and monitoring}

\section{Composition of the coordinating centre and trial steering committee $\{5 \mathrm{~d}\}$}

Principal investigator

- Design and conduct the study

- Preparation of protocol and revisions

- Organising trial steering committee meetings

- Publication of study reports

Trial steering committee

(Principal investigator and co-investigators)

- Agreement of final protocol

- Recruitment of patients

- Reviewing progress of study and if necessary agreeing changes to the protocol and/or investigators brochure to facilitate the smooth running of the study

the Principal and Co-investigators will oversee the entire trial. 
395 Composition of the data monitoring committee, its role and reporting structure $\{21 \mathrm{a}\}$

396 Data monitoring will be from a committee made up of professorial-grade staff from the department. The data monitoring committee is independent of the study organisers and have no competing interests. During the period of recruitment to the study, interim analyses will be supplied, in strict confidence, to the DMC, together with any other analyses that the committee may request. This may include analyses of data from other comparable trials.

\section{Adverse event reporting and harms $\{22\}$}

403 The research assistant will contact the participants regularly (once per week) to record any adverse events. 404 There should not be any potential harms caused to the participants. Minor adverse events such as itchiness 405 and redness of the lower limbs may be observed. However, the discomfort will disappear shortly after the 406 vibration treatment terminates. If such event occurs, it will be reported to DMEC and relevant regulatory 407 bodies as required indicating expectedness, seriousness, severity, and causality.

\section{Frequency and plans for auditing trial conduct $\{23\}$}

411 Auditing will be performed as per the Joint CUHK-NTEC guidelines.

Plans for communicating important protocol amendments to relevant parties (e.g.

414 trial participants, ethical committees) $\{25\}$

415 Principal Investigator will be responsible to update the clinical registry and ethics committee for any changes 416 in the protocol.

\section{Dissemination plans $\{31 \mathrm{a}\}$}

Journal publication and abstract presentations are planned.

\section{Discussion}

424 Diabetic foot ulcers are a massive problem affecting 110 million in China alone; it is a large social-economic burden without a good treatment option.(1-3) Whole-body vibration is a safe, non-invasive, non- 
means of non-invasive treatment of diabetic foot ulcers. The authors believe it will be embraced in diabetic

428 ulcer treatment since inexpensive vibration machines are readily available commercially and small clinics and 429 centres can easily equip themselves with one. Our study can help investigate the clinical effects of vibration 430 therapy of diabetic ulcerations and also act as a foundation for further research to investigate the mechanistic 431 pathways in which whole-body vibration modulates the body's response.

435 Protocol version01, registered on clinicaltrials.gov as NCT04275804 (19 Feb 2020). Anticipated recruitment 4361 Apr 2020 and anticipated completion 30 Mar 2023.

\section{Abbreviations}

$\begin{array}{lll}439 & \text { • } & A B I=\text { Ankle Brachial Pressure Index } \\ 440 & \text { • } & C T=\text { Computer } \text { Tomography } \\ 441 & \text { • } & \text { DM = Diabetes Mellitus } \\ 442 & \text { - } & \text { FAOS = Foot and Ankle Outcome Score } \\ 443 & \text { - } & \text { GLUT4 = glucose transporter } 4 \\ 444 & \text { - } & \text { LMHFV = Low-Magnitude High-Frequency Vibration } \\ 445 & \text { - } & \text { VEGF = vascular endothelial growth factor } \\ 446 & \text { - } & \text { Wk }=\text { Week }\end{array}$

\section{Declarations}

\section{Acknowledgements}

451 The authors acknowledge Yuen-Man Wu for her help in the paperwork for this manuscript.

\section{Authors' contributions $\{31 \mathrm{~b}\}$}

454 Authors' contributions: SKKL is the principle investigator who conceived the study and wrote the protocol. 
NCLH updated and revised the manuscript. WHC significantly contributed to the methodology and design of

456 the trial. PSHY contributed significantly to the study design and revisions of the manuscript.

457 All authors read and approved the final manuscript.

458

\section{Funding $\{\mathbf{4}\}$}

460 The project has no external funding source.

461

462 Availability of data and materials $\{29\}$

463 Any data required to support the protocol can be supplied on request.

464 Ethics approval and consent to participate $\{24\}$

465 Ethics initial approval obtained from the Joint CUHK-NTEC CREC. Ref number 2020.068

466 Written consent will be obtained from all study participants in the trial.

467

\section{Consent for publication $\{32\}$}

469 Written consent will be obtained from all study participants. This is available from the corresponding author 470 on request.

471 Competing interests $\{28\}$

472 The authors declares they have no competing interest.

\section{Author details}

475 Samuel Ka-Kin LING

476 samuel-kk-ling@alumni.cuhk.net

Dept of Orthopaedics and Traumatology, Faculty of Medicine, CUHK

Natalie Cheuk-Lam HUNG

nataliehung@cuhk.edu.hk

481 Dept of Orthopaedics and Traumatology, Faculty of Medicine, CUHK

Louis Wing-Hoi CHEUNG

484 louischeung@cuhk.edu.hk

485 Dept of Orthopaedics and Traumatology, Faculty of Medicine, CUHK

Patrick Shu-Hang YUNG

patrickyung@cuhk.edu.hk

489 Dept of Orthopaedics and Traumatology, Faculty of Medicine, CUHK 
493 Authors' information (optional)

$494 n / a$

495

496

497 References

498 1. Chan JC, Malik V, Jia W, Kadowaki T, Yajnik CS, Yoon KH, et al. Diabetes in Asia: epidemiology, risk 499 factors, and pathophysiology. Jama. 2009;301(20):2129-40.

5002 2. Chan JC, Zhang Y, Ning G. Diabetes in China: a societal solution for a personal challenge. The lancet 501 Diabetes \& endocrinology. 2014;2(12):969-79.

502 3. Chammas NK, Hill RLR, Edmonds ME. Increased Mortality in Diabetic Foot Ulcer Patients: The 503 Significance of Ulcer Type. Journal of diabetes research. 2016.

504 4. Ramachandran A, Ma RC, Snehalatha C. Diabetes in Asia. Lancet. 2010;375(9712):408-18.

505 5. Yan BP, Zhang Y, Kong AP, Luk AO, Ozaki R, Yeung R, et al. Borderline ankle-brachial index is 506 associated with increased prevalence of micro- and macrovascular complications in type 2 diabetes: A cross507 sectional analysis of 12,772 patients from the Joint Asia Diabetes Evaluation Program. Diabetes \& vascular 508 disease research. 2015;12(5):334-41.

509 6. Ma RC, Lin X, Jia W. Causes of type 2 diabetes in China. The lancet Diabetes \& endocrinology. $510 \quad 2014 ; 2(12): 980-91$.

511 7. Chan JC, Ozaki R, Luk A, Kong AP, Ma RC, Chow FC, et al. Delivery of integrated diabetes care using 512 logistics and information technology--the Joint Asia Diabetes Evaluation (JADE) program. Diabetes research 513 and clinical practice. 2014;106 Suppl 2:S295-304.

514 8. Armstrong DG, Boulton AJM, Bus SA. Diabetic Foot Ulcers and Their Recurrence. New Engl J Med. $515 \quad 2017 ; 376(24): 2367-75$.

516 9. Khalifa WA. Risk factors for diabetic foot ulcer recurrence: A prospective 2-year follow-up study in 517 Egypt. Foot (Edinb). 2018;35:11-5.

518 10. Egan AM, Dinneen SF. In-hospital metabolic regulation in patients with a diabetic foot ulcer: is it 519 worthwhile? Diabetes-Metab Res. 2016;32:297-302.

520 11. Francia P, Gulisano M, Anichini R, Seghieri G. Diabetic Foot and Exercise Therapy: Step by Step The 521 Role of Rigid Posture and Biomechanics Treatment. Current diabetes reviews. 2014;10(2):86-99. 
522 12. Zarei F, Negahdari B, Eatemadi A. Diabetic ulcer regeneration: stem cells, biomaterials, growth 523 factors. Artif Cell Nanomed B. 2018;46(1):26-32.

524 13. Margolis DJ, Kantor J, Berlin JA. Healing of diabetic neuropathic foot ulcers receiving standard 525 treatment - A meta-analysis. Diabetes care. 1999;22(5):692-5.

526 14. Leung KS, Li CY, Tse YK, Choy TK, Leung PC, Hung VW, et al. Effects of 18-month low-magnitude 527 high-frequency vibration on fall rate and fracture risks in 710 community elderly--a cluster-randomized 528 controlled trial. Osteoporosis international : a journal established as result of cooperation between the 529 European Foundation for Osteoporosis and the National Osteoporosis Foundation of the USA. $530 \quad 2014 ; 25(6): 1785-95$.

531 15. Cheung WH, Sun MH, Zheng YP, Chu WC, Leung AH, Qin L, et al. Stimulated angiogenesis for 532 fracture healing augmented by low-magnitude, high-frequency vibration in a rat model-evaluation of pulsed533 wave doppler, 3-D power Doppler ultrasonography and micro-CT microangiography. Ultrasound Med Biol. $534 \quad 2012 ; 38(12): 2120-9$.

535 16. Razzaghi R, Pourbagheri H, Momen-Heravi M, Bahmani F, Shadi J, Soleimani Z, et al. The effects of 536 vitamin D supplementation on wound healing and metabolic status in patients with diabetic foot ulcer: A 537 randomized, double-blind, placebo-controlled trial. Journal of diabetes and its complications. 2017;31(4):76653872.

539 17. Snyder R, Galiano R, Mayer P, Rogers LC, Alvarez O, Investigators ST. Diabetic foot ulcer treatment 540 with focused shockwave therapy: two multicentre, prospective, controlled, double-blinded, randomised phase $541 \quad$ III clinical trials. Journal of wound care. 2018;27(12):822-+.

542 18. Lavery L, Fulmer J, Shebetka KA, Regulski M, Vayser D, Fried D, et al. Open-label Extension Phase 543 of a Chronic Diabetic Foot Ulcer Multicenter, Controlled, Randomized Clinical Trial Using Cryopreserved 544 Placental Membrane. Wounds-a Compendium of Clinical Research and Practice. 2018;30(9):275-8.

545 19. Wong RMY, Ho WT, Tang N, Tso CY, Ng WKR, Chow SK, et al. A study protocol for a randomized 546 controlled trial evaluating vibration therapy as an intervention for postural training and fall prevention after 547 distal radius fracture in elderly patients. Trials. 2020;21(1):95.

548 20. Cheung WH, Mok HW, Qin L, Sze PC, Lee KM, Leung KS. High-frequency whole-body vibration 549 improves balancing ability in elderly women. Archives of physical medicine and rehabilitation. 2007;88(7):8525507. 
551 21. Wang J, Leung KS, Chow SK, Cheung WH. Inflammation and age-associated skeletal muscle 552 deterioration (sarcopaenia). Journal of orthopaedic translation. 2017;10:94-101.

553 22. Yu COL, Leung KS, Jiang JL, Wang TBY, Chow SKH, Cheung WH. Low-Magnitude High-Frequency 554 Vibration Accelerated the Foot Wound Healing of n5-streptozotocin-induced Diabetic Rats by Enhancing 555 Glucose Transporter 4 and Blood Microcirculation. Scientific reports. 2017;7.

556 23. Lipsky BA, Berendt AR, Cornia PB, Pile JC, Peters EJG, Armstrong DG, et al. 2012 Infectious 557 Diseases Society of America Clinical Practice Guideline for the Diagnosis and Treatment of Diabetic Foot 558 Infections. Clinical Infectious Diseases. 2012;54(12):E132-U232.

559 24. Aboyans V, Criqui MH, Abraham P, Allison MA, Creager MA, Diehm C, et al. Measurement and 560 interpretation of the ankle-brachial index: a scientific statement from the American Heart Association. 561 Circulation. 2012;126(24):2890-909.

562 25. Ling SKK, Chan V, Ho K, Ling F, Lui TH. Reliability and validity analysis of the open-source Chinese 563 Foot and Ankle Outcome Score (FAOS). Foot (Edinb). 2018;35:48-51.

56426. 